\title{
Late-onset "white cord syndrome" in an elderly patient after posterior cervical decompression and fusion: a case report
}

\author{
Ioannis Papaioannou (D) ${ }^{1} \cdot$ Thomas Repantis $^{1} \cdot$ Andreas Baikousis $^{1} \cdot$ Panagiotis Korovessis $^{1}$
}

Received: 15 December 2018 / Revised: 23 January 2019 / Accepted: 2 March 2019

(c) International Spinal Cord Society 2019

\begin{abstract}
Introduction In 2013, a rare early complication following cervical decompression the so-called "white cord syndrome" (WCS) was described for first time. This designation was given on the basis of the postoperative appearance of intramedullary hypertense areas in T2-MRI, resulting in devastating neurological damage. To our knowledge, only three cases of WCS have been published; we hereby present the fourth case, but the first one with late-onset presentation of this syndrome. Case presentation A 79-year-old male patient with Nurick grade 3 CSM was referred to our institution. He had already had a double-level C4-C6 anterior cervical decompression and fusion (ACDF) 2 years ago in another institution. The patient underwent posterior decompression from C3 to C6 plus C2-C7 lateral mass screw fusion. Within the first $24 \mathrm{~h}$ following surgery, he gradually developed C6 incomplete paraplegia (ASIA B). Cervical MRI disclosed a hypertensive signal in T2weighted sequences at C6-C7 levels and the diagnosis of WCS was suspected. Revision surgery was made $30 \mathrm{~h}$ following our first surgery, with wider posterior decompression accompanied by intravenous methylprednisolone. The patient's neurologic status was improved, but the final neurologic outcome was worse (Nurick 4) than the preoperative status and subsequently did not change at all.

Discussion To the best of our knowledge, this is the first report of a late-onset WCS and the fourth case of WCS per se. Spine surgeons should be aware of this rare but serious complication. We highlight possible risk factors and review the literature on the hypotheses about the pathophysiology of WCS.
\end{abstract}

\section{Introduction}

Cervical spondylotic myelopathy (CSM) describes a functional disturbance in the spinal cord due to degenerative changes within the cervical spine [1]. Anterior versus posterior approach to address CSM is still under debate. The literature suggests that both approaches have similar functional outcomes and similar rates of complications [2]. The posterior approach, a frequently performed technique for CSM, includes mainly laminectomy and fusion to improve clinical manifestations and prevent further neurologic deterioration. Neurological deficits after posterior cervical decompression and fusion (PCDF) are very rare, with incidence approximately $0.18 \%$ [3]. The main causes of

Ioannis Papaioannou

john-pane1984@hotmail.com

1 Orthopedic Department of General Hospital of Patras Spine and Trauma Unit, Patras, Greece neurologic deterioration are hematomas and neurovascular injuries. C5 nerve root palsy is a well-known devastating complication after posterior decompression, with an incidence of 5.9\% [4]. A very rare, relatively recently described entity, the so-called "white cord syndrome" (WCS) was first described in 2013 [5]. This designation was given because of the postoperative appearance of intramedullary hypertense areas in T2-weighted magnetic resonance imaging (MRI), resulting in devastating neurological damage. Since then, two new cases (one after anterior and one after posterior approach) of WCS with intraoperative onset of the neurological deficit have been recorded $[6,7]$. We report the fourth case of this rare syndrome, but in contrast to the previously reported three cases, manifestation of the neurologic deficit in our case appeared delayed and not intraoperatively. The etiology of this complication is still unknown, and the prevailing hypothesis is reperfusion injury in a severe and chronically damaged spinal cord after anterior or posterior decompression.

By this case report, we support the hypothesis that the reperfusion injury can also be subacute (until $24 \mathrm{~h}$ following 
decompression) [8], especially in an elderly population with atherosclerotic vessels, chronic hypertension, and other comorbidities [9], rather than being exclusively acute as in the three previous cases. Furthermore, delayed decompression exacerbates ischemia-reperfusion in cervical compressive myelopathy [10]. Spine surgeons should be aware of this extremely rare complication, not only intraoperatively but also postoperatively, in order to recognize this potential disastrous complication early on.

\section{Case presentation}

A 79-year-old male patient, who had suffered from gait disturbances and neck pain for at least 2 years, was referred to our department for CSM. He had already had a doublelevel anterior cervical decompression and fusion (ACDF) with anterior plate and cage 2 years before in another institution (Fig. 1), although the symptoms didn't recede and became worse. Physical examination on admission revealed grip weakness of the right hand, mild weakness of the right leg, numbness, clonus, hypertonia of lower extremities, and positive Hoffman and Lhermitte sign, while no sensory deficits were disclosed. On this initial admission he was assessed as Nurick grade 3 and MRI revealed a multilevel significant posterior stenosis from the $\mathrm{C} 4$ to the C6 vertebrae (Fig. 2), with no sign of spinal cord ischemia. His medical history disclosed hypertension and atrial fibrillation and he was receiving irbesartan (angiotensin receptor blocker) and apixaban (direct selective factor Xa inhibitor). Based on the clinical and radiological findings (compression onto the posterior cervical spinal cord) and with the limitation of the previous surgical intervention, he underwent posterior decompression from $\mathrm{C} 3$ to $\mathrm{C} 6$ and posterior fusion with lateral mass screws from $\mathrm{C} 2$ to $\mathrm{C} 7$
(Fig. 3). The intraoperative neuromonitoring showed no intraoperative neurologic worsening or deviations from the preoperative baseline. No surgical nor anesthesiologic complications were observed. Our patient returned to our clinic with no sign of neurological deficit but, to the contrary, with mild improvement of his preoperative symptoms. Twenty-four hours postoperatively, the patient complained of inability to move both his legs and arms. After a detailed physical exanimation, a diagnosis of C6-level incomplete paraplegia (ASIA B) was settled. Urgent brain and cervical computed tomography scans were made that however disclosed no signs of bony compression of the cord or any screw malposition. Immediately, a high dose of steroid was administrated based on NASCIS 3 protocol for $48 \mathrm{~h}$. Unfortunately, we did not have the opportunity to perform MRI in this public hospital and we decided not to squander valuable time with a referral of our patient to another facility. Thus, the patient was urgently returned to the operating room with the suspicion of an intracanal hematoma compressing the already decompressed spinal cord. Intraoperatively, we found no hematoma and no cerebrospinal fluid leakage. During this second surgery, we extended our decompression to provide more space to the cord for possible recovery. Postoperatively, we performed cervical spine MRI that disclosed a hypertense signal in T2weighted sequences at C6-C7 vertebrae (Figs. 4 and 5), just below the lowest level of our decompression. Based on the clinical findings and the myelomalacia at the MRI, the diagnosis of the WCS was settled and steroid administration was held due to reperfusion spinal cord injury. Our patient $48 \mathrm{~h}$ later had a gradual neurologic improvement, mostly in the upper extremities. Physical examination on day 3 after the second surgery revealed 3/5 strength in the right upper extremity on the Medical Research Council (MRC) Scale, 4/5 strength (MRC) in the left upper extremity, 2/5 strength
Fig. 1 Sagittal computed tomography image on admission of the patient to our department. We discern the plate and the cage after the anterior approach and decompression 2 years before at another institution

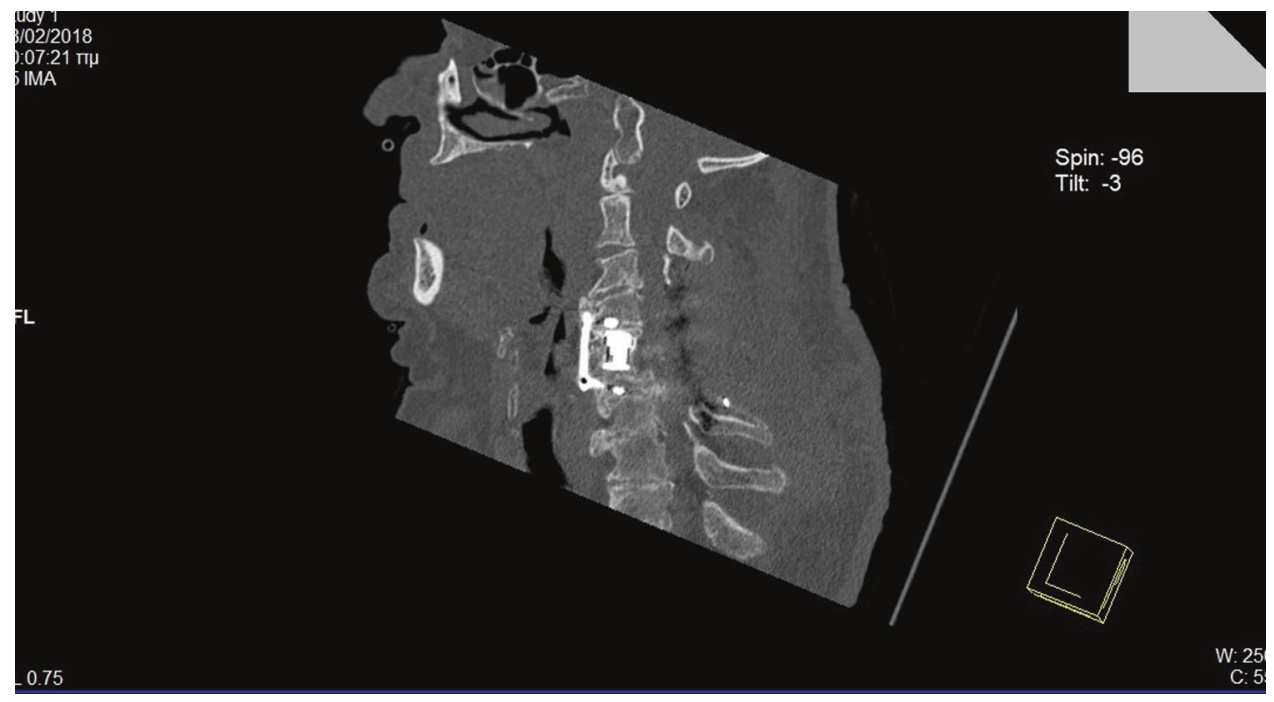


Fig. 2 Sagittal magnetic resonance imaging image of the cervical spine showing the anterior plate and the cage on admission. This image demonstrates the significant compression of the cord at C4-C6 vertebrae level (blue arrow)
Fig. 3 Sagittal computed tomography image after the first decompression surgery to our department demonstrates the posterior decompression and fusion from $\mathrm{C} 2$ to $\mathrm{C} 7$, while no screw malposition was observed

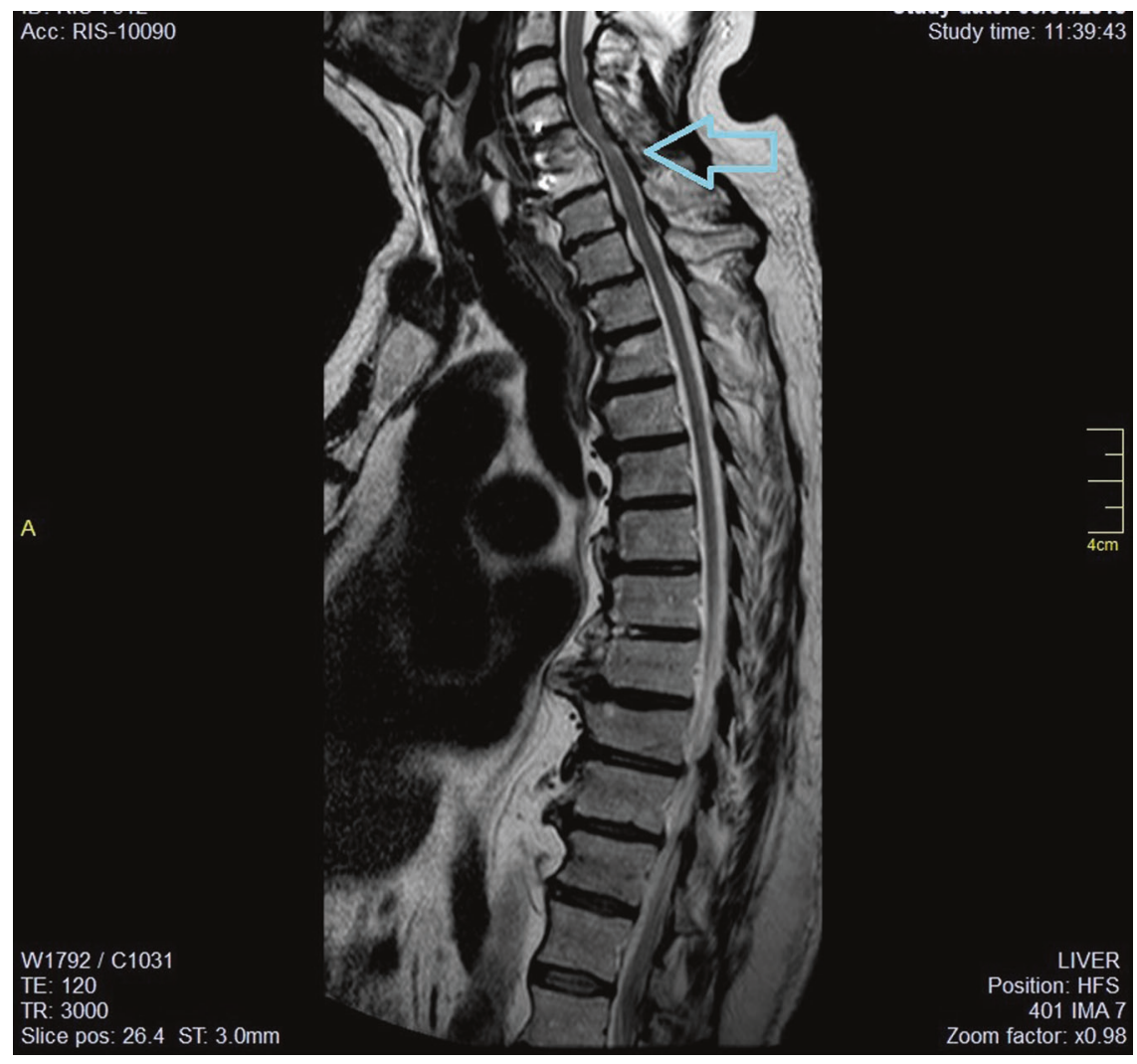

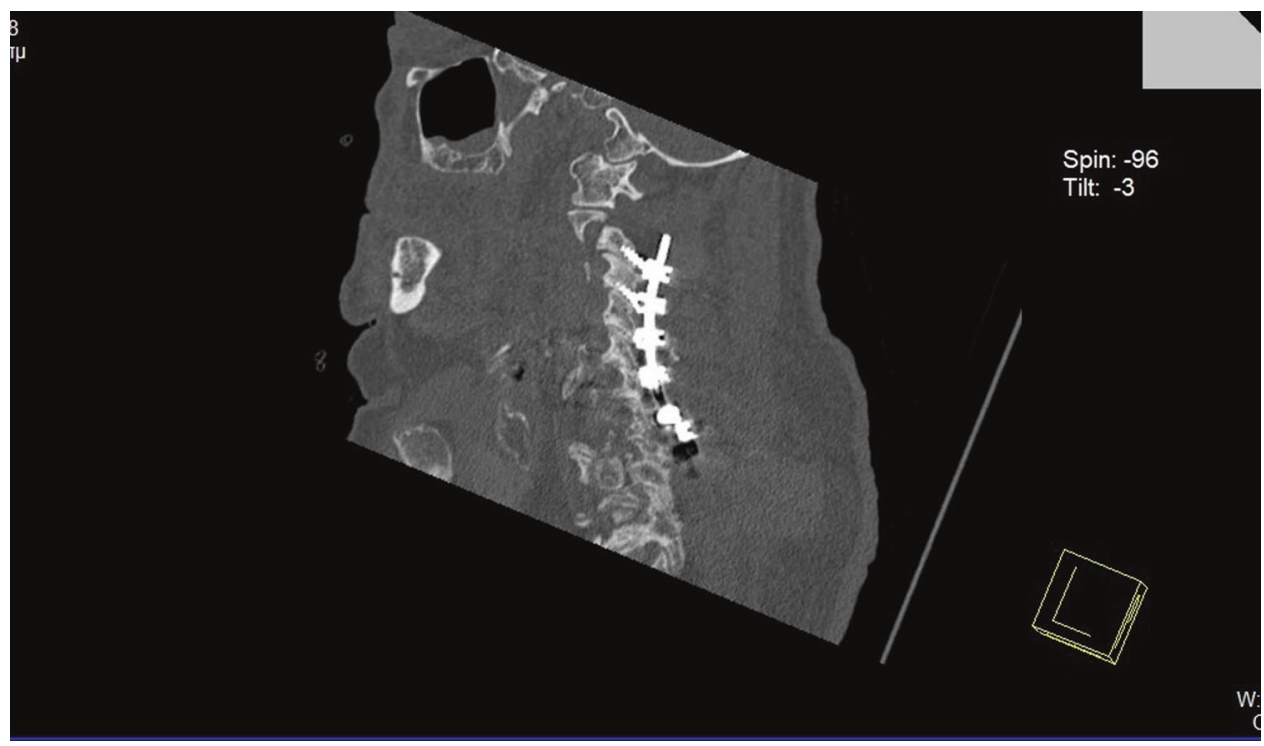

(MRC) in the right leg, and 3/5 strength (MRC) in the left leg. Our patient started physical therapy on day 4 , but he was ambulated only with excessive assistance from the physiotherapists. Nurick grade 4 was assessed and our patient was discharged on day 9 to an inpatient rehabilitation center. At the 6, 12, and 18-month follow-up, there were no remarkable changes in the functional status of our patient.

\section{Discussion}

WCS is a very rare surgical complication, characterized by an ischemic lesion of the spinal cord after anterior or posterior decompression of the cervical spine [5]. This designation was given due to postoperative appearance of intramedullary hypertense areas in T2-weighted magnetic 
Fig. 4 Postoperative sagittal T2 magnetic resonance imaging image demonstrates a wide posterior decompression of the cervical spinal cord. A huge intramedullary hypertense area, exactly below the C6 vertebrae (green arrow) after the second surgery to our department approximately $24-28 \mathrm{~h}$ after the first surgery

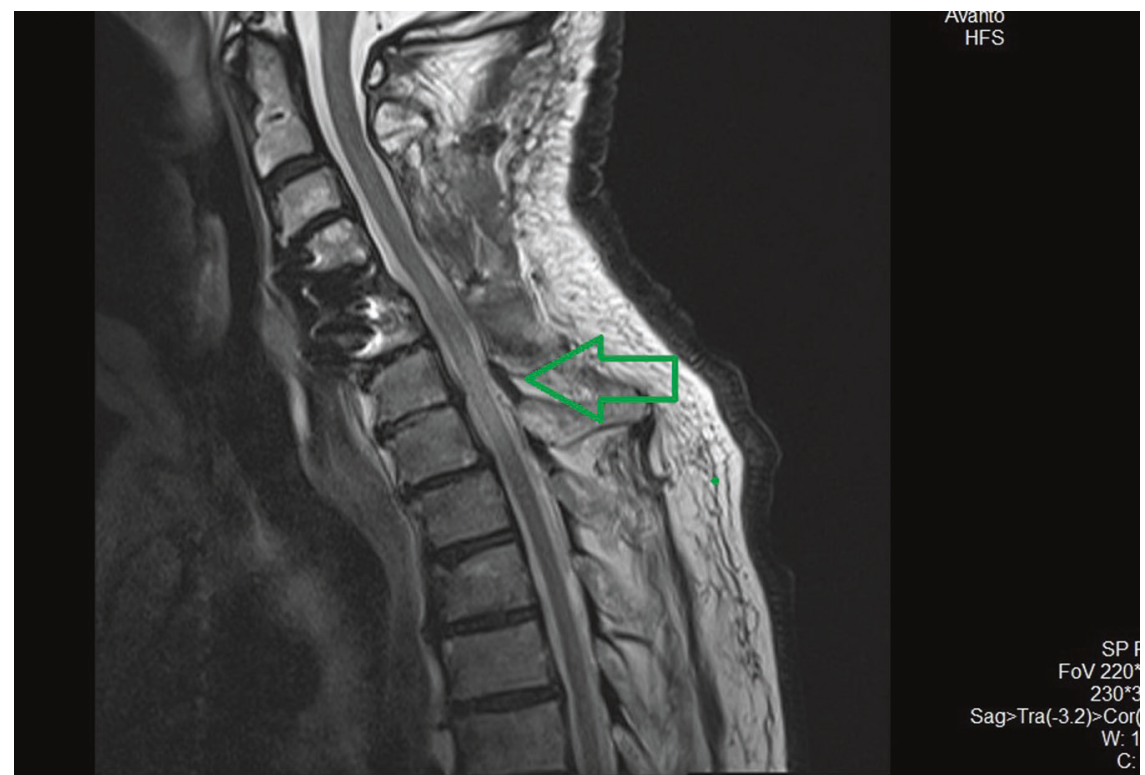

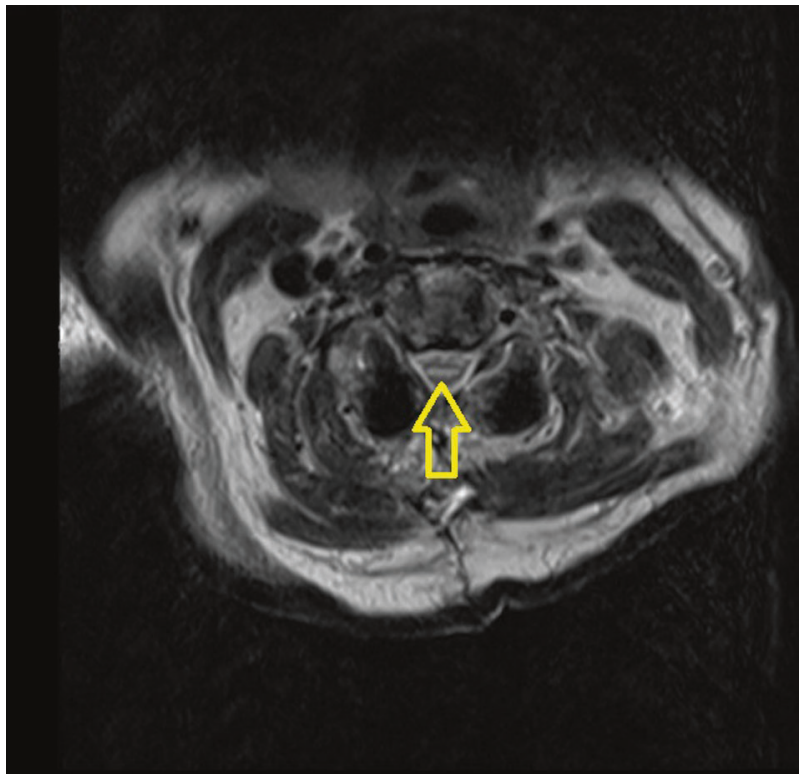

Fig. 5 Axial T2 magnetic resonance imaging image after the second surgery demonstrates clearly the intramedullary hypertense area (yellow arrow), highlighting the presence of the WCS

resonance (MR) images, resulting in devastating neurological damage [5]. This syndrome has already been described three times (two cases after anterior approach and one case after posterior decompression) in the literature (Table 1) [57]. In all previous cases the neurologic deficit occurred intraoperatively, whereas in our case the deficit first became manifest $24 \mathrm{~h}$ postoperatively. All patients with WCS, including our case, were treated with high-dose steroid protocols and showed remarkable improvement in symptoms. Surgical revision and further decompression were decided upon in three of four cases with WCS, including our patient. In all cases, the final outcome was worse than the preoperative status and this made the complication all the more devastating, for patients and surgeons alike.

Differential diagnosis of WCS includes iatrogenic trauma resulting in cerebrospinal fluid leakage and pseudo meningocele, cerebrovascular insult, and inception of an undiagnosed demyelinating disease [7]. In our case, we had excluded all these possible diagnoses, because we didn't find any hematoma or cerebrospinal fluid leakage, while the brain computed tomography and MRI scan were normal.

In 2014, a cohort study described the incidence of immediate spinal cord deficits and delayed onset of nerve root deficits after laminoplasty and the correlation of these deficits with the pertinent MR images [11]. A $6.1 \%$ incidence of postoperative abnormal expansion of the T2 highsignal intensity area was reported, of which $25 \%$ (3/12) were asymptomatic. In all 114 cases, a high-signal intensity area in the spinal cord was present at the most stenotic level on preoperative T2-weighted MRI. No patient with postoperative C5 and C6 palsies showed abnormal spreading of the high intensity area, which supports the theory of rootimpairment as the cause of this proximal type of palsy [4]. These deficits with the T2 high signal expansion were more proximal, distal, and diffuse upper motor paresis. None of the patients in this study showed the deterioration of lower motor function that our patient did, but the pathophysiology of all these lesions may share common pathways with the "WCS," since the MRI findings are similar. It is worthwhile noting that the majority $(8 / 9)$ of the patients in this study with the $\mathrm{T} 2$ expansion and the neurological deficit were male, with a mean age of 65.6 years. The patients reported with the "WCS" are all male (4/4) with a mean age of 67.5 years. Unfortunately, the medical history of the 3 other 


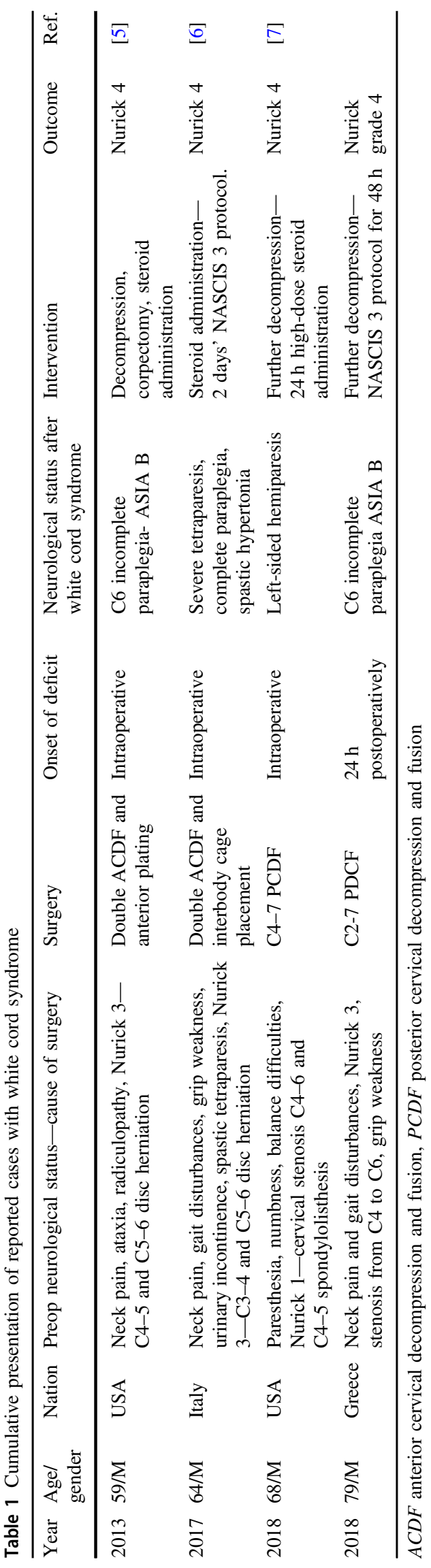

cases with the "WCS" were not included in their report by the authors. More recently, papers published in the literature have revealed new neurological deficits after anterior or posterior approach to surgery, including root palsy (except C5-C6) and worsening of the preexisting myelopathy $[2,12,13]$. In these studies, the authors didn't perform MRI on the neurologically impaired patients to correlate the deficit with the MR images. The mechanism of the WCS is still unclear and very little is known. To best of our knowledge secondary ischemia is playing a crucial role. We believe that WCS and other neurological deficits after decompression surgery, except C5-C6 nerve palsies, refer at the same mechanism damaging the spinal cord. Although, the severity of the deficit, the onset of the neurological deterioration and the development of the white cord area in T2-weighted images varies. Moreover, most infarctions result more or less in a "white cord area" in T2weighted images.

The main hypothesis of the WCS is the ischemiareperfusion injury [5] while a simpler mechanism is the direct trauma from the rush of the blood flow after the reperfusion. The sudden cord expansion due to the decompression of the chronically ischemic tissue acts as a theft of blood flow from bottom (C6-C7, less compressed area) to top (C3-C5, most compressed area). Chronic ischemia is characterized by increased quantity of nuclear factor kappa-light-chain-enhancer of activated B cells (nuclear factor- $\mathrm{KB}(\mathrm{NF}-\mathrm{\kappa B})$ ) gene products, such as tumor necrosis factor- $\alpha$ (TNF-alpha), interleukin (IL)-1 $\beta$, or IL-6, resulting in excessive oxidative stress [14]. Perhaps the reperfusion leads to disruption of the blood-brain barrier (BBB) or of blood-spinal cord barrier (BSB) $[15,16]$. Such a breakdown of the BBB may lead to increased permeability of the inflammatory factors [17], resulting in neuronal apoptosis [18].

The consequence is that significant amounts of oxygen free radical oxygen species (oxidants) are generated during the reperfusion, and oxidative stress plays a crucial role in neural elements damage. In addition to oxidizing macromolecules, leading to cell injury, oxidants are also involved in cell death/survival signal pathways and cause mitochondrial dysfunction [19]. Especially, the free radical oxygen species damage the phospholipid-rich membrane of neurons. Thus, recently it has been proposed that potent antioxidants may also play a role in the management of spinal cord ischemic/reperfusion injury [14]. Our findings should assist in the development of novel therapeutic strategies for Spinal Cord Injury Research Evidence. The improvement of the neurological status after high dose of corticosteroid administration in all abovementioned cases strengthens the hypothesis of the oxidative stress. Furthermore, a recent experimental (mice model) study suggests perioperative methylprednisolone administration to 
reduce neurological complications after decompression surgery [20]. Steroids upregulate anti-inflammatory factors and decreases oxidative stress, enhancing endogenous cell survival in animal models of spinal cord injury. Methylprednisolone reduces edema, prevents intracellular potassium depletion, and inhibits lipid peroxidation [21].

We postulate that the delay of the clinical manifestation in our elderly male patient was due to the endothelial damage and atherosclerosis caused by chronic hypertension. There have been reports of a decline in the nitric oxide (NO) precursors such as aspartic acid, citrulline, and arginine as well as an age-dependent decrease in endotheliumdependent relaxation, which is thought to be mediated by NO [22]. This led to subacute reperfusion to the compressed spinal cord area, resulting to this late-onset case of WCS. Unfortunately, in our hospital facilities we don't have the potentiality to measure any biomarker for the oxidative stress. As we mentioned above, chronic ischemia and reperfusion injury generate reactive oxygen species (ROS) and results in breakdown of the BBB. Furthermore, elderly people are subject to a physiological condition called "inflamm-aging." This condition is characterized by an imbalance between antioxidants and ROS, with the ROS predominating. These increased amounts of ROS can cause cell death to neurons [23]. Until now, neither risk factors nor prognostic factors had been suggested for the WCS. According to the above-mentioned hypothesis and based on experimental and epidemiologic studies the current literature suggests that the increased age of the patient, any comorbidities, and the chronic compression of the cord are potential risk factors and should be so identified. Specifically, recent experimental studies in mice models suggest that delayed decompression can exacerbate reperfusion injury and is associated with ongoing enhanced levels of cytokine expression, microglial activation, and astrogliosis [10], while early decompression inhibits the expression of TNF- $\alpha$ [24]. Furthermore, an extensive epidemiologic international study, noticed that older patients with comorbidities accompanied with severe and long-term compression of the cervical spinal cord undergoing surgical decompression, had a poorer functional outcome according to modified Japanese Orthopaedic Association score [9]. Although, well-designed human studies are mandatory.

WCS is a rare but ravaging complication after ACDF or PCDF of which surgeons should be aware. To the best of our knowledge, this is the first report of a late-onset presentation of the syndrome. Anesthesiologists and surgeons should be aware that propofol administration can potentially prevent BSB disruption in case of ischemia-reperfusion injury, by inhibiting the NF-kB signaling pathway [25]. The beneficial effect of perioperative administration of methylprednisolone should be investigated by human studies and the adverse effects should also be taken into consideration
[20]. We therefore encourage surgeons to include this complication in the written consent before spinal surgery. Spine surgeons should be alert not only intraoperatively but also postoperatively, in order to notice and diagnose this disastrous complication as soon as possible. Further studies are also mandated, to investigate the exact mechanism of the syndrome, in order to enable the timely treatmentperhaps even the prevention-of this rare but devastating complication.

\section{Compliance with ethical standards}

Conflict of interest The authors declare that they have no conflict of interest.

Publisher's note: Springer Nature remains neutral with regard to jurisdictional claims in published maps and institutional affiliations.

\section{References}

1. Young WF. Cervical spondylotic myelopathy: a common cause of spinal cord dysfunction in older persons. Am Fam Physician. 2000;62:1064-70, 1073.

2. Fehlings MG, Barry S, Kopjar B, Yoon ST, Arnold P, Massicotte $\mathrm{EM}$, et al. Anterior versus posterior surgical approaches to treat cervical spondylotic myelopathy. Spine (Phila Pa 1976). 2013;38:2247-52.

3. Currier BL. Neurological complications of cervical spine surgery: C5 palsy and intraoperative monitoring. Spine (Phila Pa 1976). 2012;37:E328-34.

4. Cuellar J, Passias P. Cervical spondylotic myelopathy a review of clinical diagnosis and treatment. Bull Hosp Jt Dis. 2017;75:21-9.

5. Chin KR, Seale J, Cumming V. "White cord syndrome" of acute tetraplegia after anterior cervical decompression and fusion for chronic spinal cord compression: a case report. Case Rep Orthop. 2013;2013:697918.

6. Giammalva GR, Maugeri R, Graziano F, Gulì C, Giugno A, Basile $\mathrm{L}$, et al. White cord syndrome after non-contiguous doublelevel anterior cervical decompression and fusion (ACDF): A "no reflow phenomenon"? Interdiscip Neurosurg. 2017;7:47-9.

7. Antwi P, Grant R, Kuzmik G, Abbed K. "White cord syndrome" of acute hemiparesis after posterior cervical decompression and fusion for chronic cervical stenosis. World Neurosurg. 2018;113:33-6.

8. Karadimas SK, Laliberte AM, Tetreault L, Chung YS, Arnold P, Foltz WD, et al. Riluzole blocks perioperative ischemiareperfusion injury and enhances postdecompression outcomes in cervical spondylotic myelopathy. Sci Transl Med. 2015;7:316ra194.

9. Tetreault L, Kopjar B, Côté P, Arnold P, Fehlings MG. A clinical prediction rule for functional outcomes in patients undergoing surgery for degenerative cervical myelopathy: analysis of an international prospective multicenter data set of 757 subjects. J Bone Jt Surg Am. 2015;97:2038-46.

10. Vidal PM, Karadimas SK, Ulndreaj A, Laliberte AM, Tetreault L, Forner S, et al. Delayed decompression exacerbates ischemiareperfusion injury in cervical compressive myelopathy. JCI Insight. 2017;2.

11. Seichi A, Takeshita K, Kawaguchi H, Nakajima S, Akune T, Nakamura K. Postoperative expansion of intramedullary high- 
intensity areas on T2-weighted magnetic resonance imaging after cervical laminoplasty. Spine (Phila Pa 1976). 2004;29:1478-82.

12. Fountas KN, Kapsalaki EZ, Nikolakakos LG, Smisson HF, Johnston KW, Grigorian AA, et al. Anterior cervical discectomy and fusion associated complications. Spine (Phila Pa 1976). 2007;32:2310-7.

13. Fehlings MG, Smith JS, Kopjar B, Arnold PM, Yoon ST, Vaccaro $\mathrm{AR}$, et al. Perioperative and delayed complications associated with the surgical treatment of cervical spondylotic myelopathy based on 302 patients from the AOSpine North America Cervical Spondylotic Myelopathy Study. J Neurosurg Spine. 2012;16:425-32.

14. Shirley R, Ord ENJ, Work LM. Oxidative stress and the use of antioxidants in stroke. Antioxidants (Basel). 2014;3:472-501.

15. Bai J, Lyden PD. Revisiting cerebral postischemic reperfusion injury: new insights in understanding reperfusion failure, hemorrhage, and edema. Int J Stroke. 2015;10:143-52.

16. Lee JY, Choi HY, Ahn H-J, Ju BG, Yune TY. Matrix metalloproteinase-3 promotes early blood-spinal cord barrier disruption and hemorrhage and impairs long-term neurological recovery after spinal cord injury. Am J Pathol. 2014;184:2985-3000.

17. Pan W, Banks WA, Kastin AJ. Blood-brain barrier permeability to ebiratide and TNF in acute spinal cord injury. Exp Neurol. 1997;146:367-73.

18. Sharma HS. Pathophysiology of blood-spinal cord barrier in traumatic injury and repair. Curr Pharm Des. 2005;11:1353-89.
19. Chen H, Yoshioka H, Kim GS, Jung JE, Okami N, Sakata H, et al. Oxidative stress in ischemic brain damage: mechanisms of cell death and potential molecular targets for neuroprotection. Antioxid Redox Signal. 2011;14:1505-17.

20. Vidal PM, Ulndreaj A, Badner A, Hong J, Fehlings MG. Methylprednisolone treatment enhances early recovery following surgical decompression for degenerative cervical myelopathy without compromise to the systemic immune system. J Neuroinflamm. 2018;15:222.

21. Wilson JR, Forgione N, Fehlings MG. Emerging therapies for acute traumatic spinal cord injury. Can Med Assoc J. 2013;185:485-92.

22. Strolin Benedetti M, Dostert P, Marrari P, Cini M. Effect of ageing on tissue levels of amino acids involved in the nitric oxide pathway in rat brain. J Neural Transm Gen Sect. 1993;94:21-30.

23. Fuente M, Miquel J. An update of the oxidation-inflammation theory of aging: the involvement of the immune system in oxiinflamm-aging. Curr Pharm Des. 2009;15:3003-26.

24. Xie J-B, Zhang X, Li Q-H, Xu Z-J. Inhibition of inflammatory cytokines after early decompression may mediate recovery of neurological function in rats with spinal cord injury. Neural Regen Res. 2015;10:219-24.

25. Xie L-J, Huang J-X, Yang J, Yuan F, Zhang S-S, Yu Q-J, et al. Propofol protects against blood-spinal cord barrier disruption induced by ischemia/reperfusion injury. Neural Regen Res. 2017;12:125-32. 